\title{
Thermodynamic evaluation and chemical vapor transport of few-layer $\mathrm{WTe}_{2}$
}

Felix Hansen', Martin Wels², Samuel Froeschke', Alexey Popov' ${ }^{1}$, Daniel Wolf ${ }^{1}$, Bernd Büchner ${ }^{1,3}$, Peer Schmidt ${ }^{2}$, Silke Hampel ${ }^{1}$

${ }^{1}$ Leibniz Institute for Solid State and Materials Research Dresden, Helmholtzstraße 20, 01069 Dresden, Germany

${ }^{2}$ Brandenburg University of Technology Cottbus - Senftenberg, Universitätsplatz 1, o1968 Senftenberg, Germany

3 Technische Universität Dresden, Helmholtzstr. 10, 01069, Dresden, Germany

- Supporting Information - 


\section{Supporting Information.}

Thermodynamic data used in all simulations and models mentioned in the publication "Thermodynamic evaluation and chemical vapor transport of fewlayer $\mathrm{WTe}_{2}$ ".

Table 1. Condensed species used in FactSage $7.3^{1}$

\begin{tabular}{|c|c|c|c|c|c|c|c|c|c|c|c|c|}
\hline \multirow{3}{*}{$\begin{array}{c}\text { formula } \\
\text { W }\end{array}$} & \multirow{3}{*}{$\begin{array}{c}\text { state } \\
\mathrm{s} \\
\end{array}$} & \multirow{3}{*}{$\begin{array}{c}\text { source } \\
\text { SGPS }\end{array}$} & \multirow{3}{*}{$\begin{array}{c}\begin{array}{c}H(298 \mathrm{~K}) \\
\text { in } \mathrm{J} \cdot \mathrm{mol}^{-1}\end{array} \\
0.0\end{array}$} & \multirow{3}{*}{$\begin{array}{c}\begin{array}{c}\mathrm{S}(298 \mathrm{~K}) \\
\text { in } \mathrm{J} \cdot \mathrm{K}^{-1} \cdot \mathrm{mol}^{-1}\end{array} \\
32.618\end{array}$} & \multirow{2}{*}{\multicolumn{2}{|c|}{$C_{p, \mathrm{~m}}$-range }} & \multicolumn{6}{|c|}{$C_{p, \mathrm{~m}}=\left(a \cdot T^{0}+b \cdot T^{1}+c \cdot T^{-2}+d \cdot T^{2}+e \cdot T^{-0.5}+f \cdot T^{3}\right)$ in J.mol $\mathrm{mol}^{-1} \cdot \mathrm{K}^{-1}$} \\
\hline & & & & & & & \multirow{2}{*}{$\begin{array}{c}a \cdot T^{0} \\
24.100\end{array}$} & \multirow{2}{*}{$\begin{array}{c}\boldsymbol{b} \cdot \boldsymbol{T}^{\mathbf{1}} \\
3.872 \mathrm{E}-3\end{array}$} & \multirow{2}{*}{$\begin{array}{c}c \cdot T^{-2} \\
-8.900 \mathrm{E}+4\end{array}$} & \multirow{2}{*}{$\begin{array}{c}\boldsymbol{d} \cdot \boldsymbol{T}^{\mathbf{2}} \\
-1.242 \mathrm{E}-6\end{array}$} & \multirow{2}{*}{$\begin{array}{l}e \cdot T^{-0.5} \\
0.000\end{array}$} & \multirow{2}{*}{$\begin{array}{c}f \cdot T^{3} \\
6.396 \mathrm{E}-10\end{array}$} \\
\hline & & & & & 298.15 & 3695 & & & & & & \\
\hline $\mathrm{WTe}_{2}$ & $\mathbf{s}$ & estimated & -26500.0 & 132.750 & 298.15 & 1501 & 84.467 & 1.079E-3 & $-7.070 \mathrm{E}+5$ & $0.000 \mathrm{E}+0$ & 0.000 & $0.000 \mathrm{E}+0$ \\
\hline \multirow[t]{4}{*}{ Te } & I & FactPS & & & 298.15 & 626.49 & 126.318 & $-4.439 \mathrm{E}-1$ & $-1.656 \mathrm{E}+6$ & $5.652 \mathrm{E}-4$ & 0.000 & $0.000 \mathrm{E}+0$ \\
\hline & & & & & 626.49 & 722.66 & 7196.409 & $-1.420 E+1$ & $-5.161 E+8$ & $7.842 \mathrm{E}-3$ & 0.000 & $0.000 E+0$ \\
\hline & & & & & 722.66 & 1150 & -202.743 & $2.840 \mathrm{E}-1$ & $4.848 \mathrm{E}+7$ & $-9.678 E-5$ & 0.000 & $0.000 \mathrm{E}+0$ \\
\hline & & & & & 1150 & 1600 & 32.560 & $0.000 E+0$ & $0.000 E+0$ & $0.000 E+0$ & 0.000 & $0.000 \mathrm{E}+0$ \\
\hline \multirow[t]{4}{*}{ Te } & $\mathbf{s}$ & FactPS & 0.0 & 49.221 & 298.15 & 626.49 & 126.318 & $-4.439 \mathrm{E}-1$ & $-1.656 \mathrm{E}+6$ & $5.652 \mathrm{E}-4$ & 0.000 & $0.000 \mathrm{E}+0$ \\
\hline & & & & & 626.49 & 722.66 & 7196.409 & $-1.420 \mathrm{E}+1$ & $-5.161 \mathrm{E}+8$ & $7.842 \mathrm{E}-3$ & 0.000 & $0.000 E+0$ \\
\hline & & & & & 722.66 & 1150 & -202.743 & $2.840 \mathrm{E}-1$ & $4.848 E+7$ & $-9.678 E-5$ & 0.000 & $0.000 \mathrm{E}+0$ \\
\hline & & & & & 1150 & 1600 & 32.560 & $0.000 \mathrm{E}+0$ & $0.000 \mathrm{E}+0$ & $0.000 \mathrm{E}+0$ & 0.000 & $0.000 \mathrm{E}+0$ \\
\hline $\mathrm{WOBr}_{2}$ & $\mathbf{s}$ & $\begin{array}{l}H \& S \text { from }^{2} \\
C_{\mathrm{p}} \text { estimated }\end{array}$ & -489071.9 & 150.599 & 298.15 & 6000 & 94.935 & $0.000 \mathrm{E}+0$ & $0.000 E+0$ & $0.000 \mathrm{E}+0$ & 0.000 & $0.000 \mathrm{E}+0$ \\
\hline $\mathbf{W}$ & I & SGPS & 52313.7 & 46.776 & 298.15 & 3695 & 24.100 & $3.872 \mathrm{E}-3$ & $-8.900 \mathrm{E}+4$ & $-1.242 \mathrm{E}-6$ & 0.000 & $6.396 \mathrm{E}-10$ \\
\hline $\mathrm{WOBr}_{3}$ & $\mathbf{s}$ & $\begin{array}{l}H \& S \text { from }^{2} \\
C_{\mathrm{p}} \text { estimated }\end{array}$ & -557325.5 & 192.472 & 298.15 & 6000 & 119.922 & $0.000 \mathrm{E}+0$ & $0.000 E+0$ & $0.000 E+0$ & 0.000 & $0.000 \mathrm{E}+0$ \\
\hline $\mathrm{Te}_{2} \mathrm{Br}$ & $\mathbf{s}$ & 3 & -48187.1 & 147.432 & 298.15 & 6000 & 62.057 & $5.086 \mathrm{E}-2$ & $0.000 \mathrm{E}+0$ & $0.000 E+0$ & 0.000 & $0.000 \mathrm{E}+0$ \\
\hline $\mathrm{WOBr}_{4}$ & $\mathbf{s}$ & 4 & -588308.1 & 259.425 & 298.15 & 6000 & 133.892 & $-1.046 \mathrm{E}-2$ & $0.000 \mathrm{E}+0$ & $0.000 \mathrm{E}+0$ & 0.000 & $0.000 \mathrm{E}+0$ \\
\hline $\mathrm{WBr}_{4}$ & $\mathbf{s}$ & FactPS & -265000.0 & 228.000 & 298.15 & 1000 & 123.140 & $2.972 \mathrm{E}-2$ & $-1.532 \mathrm{E}+2$ & $4.622 \mathrm{E}-9$ & 0.000 & $0.000 \mathrm{E}+0$ \\
\hline $\mathrm{WO}_{2}$ & $s$ & FactPS & -589693.0 & 50.528 & 298.15 & 1300 & 117.792 & $-1.266 \mathrm{E}-2$ & $-2.024 E+5$ & $3.962 \mathrm{E}-6$ & -973.278 & $0.000 E+0$ \\
\hline \multirow[t]{2}{*}{$\mathrm{WBr}_{5}$} & $\mathbf{s}$ & FactPS & -311708.0 & 271.960 & 298.15 & 1000 & 125.863 & $1.001 \mathrm{E}-1$ & $-2.223 E+4$ & $0.000 \mathrm{E}+0$ & 0.000 & $0.000 \mathrm{E}+0$ \\
\hline & & & & & 1000 & 1001 & 136.000 & $0.000 \mathrm{E}+0$ & $0.000 \mathrm{E}+0$ & $0.000 \mathrm{E}+0$ & 0.000 & $0.000 \mathrm{E}+0$ \\
\hline
\end{tabular}



Table 2. Gaseous species used in FactSage $7.3^{1}$

\begin{tabular}{|c|c|c|c|c|c|c|c|c|c|c|c|c|c|}
\hline \multirow{3}{*}{$\begin{array}{c}\text { formula } \\
\mathrm{Te}_{2}\end{array}$} & \multirow{3}{*}{$\begin{array}{c}\text { state } \\
\mathrm{g}\end{array}$} & \multirow{3}{*}{$\begin{array}{l}\text { source } \\
\text { FactPS }\end{array}$} & \multirow{3}{*}{$\begin{array}{c}\begin{array}{c}H(298 K) \\
\text { in J·mol }\end{array} \\
162063.0\end{array}$} & \multirow{3}{*}{$\begin{array}{c}\begin{array}{c}S(298 \mathrm{~K}) \\
\text { in } \mathrm{J} \cdot \mathrm{K}^{-1} \cdot \mathrm{mol}^{-1}\end{array} \\
258.944 \\
\end{array}$} & \multirow{2}{*}{\multicolumn{2}{|c|}{$C_{p, \mathrm{~m}}$-range }} & \multicolumn{7}{|c|}{$C_{p, \mathrm{~m}}=\left(a \cdot T^{0}+b \cdot T^{1}+c \cdot T^{-2}+d \cdot T^{2}+e \cdot T^{-0.5}+f \cdot T^{3}\right)$ in $\cdot \cdot \mathrm{mol}^{-1} \cdot \mathrm{K}^{-1}$} \\
\hline & & & & & & & \multirow{2}{*}{$\begin{array}{c}a \cdot T^{0} \\
35.701\end{array}$} & \multirow{2}{*}{\begin{tabular}{|c|}
$\boldsymbol{b} \cdot \boldsymbol{T}^{\mathbf{1}}$ \\
$-1.507 \mathrm{E}-3$ \\
\end{tabular}} & \multirow{2}{*}{\begin{tabular}{c|}
$c \cdot T^{-2}$ \\
$0.000 \mathrm{E}+0$
\end{tabular}} & \multirow{2}{*}{$\begin{array}{c}d \cdot T^{2} \\
1.635 \mathrm{E}-5\end{array}$} & \multirow{2}{*}{\begin{tabular}{|l|}
$e \cdot T^{-0.5}$ \\
0.000
\end{tabular}} & \multirow{2}{*}{\begin{tabular}{|c|}
$f \cdot T^{-1}$ \\
0.000
\end{tabular}} & \multirow{2}{*}{\begin{tabular}{|c|}
$g \cdot T^{\mathbf{3}}$ \\
$0.000 \mathrm{E}+0$
\end{tabular}} \\
\hline & & & & & 298.15 & 500 & & & & & & & \\
\hline & & FactPS & & & 500 & 1000 & 21.905 & $4.038 \mathrm{E}-2$ & $4.005 E+5$ & $-1.864 \mathrm{E}-5$ & 0.000 & 0.000 & $0.000 \mathrm{E}+0$ \\
\hline $\mathbf{N}_{2}$ & $\mathbf{g}$ & FactPS & 0.0 & 191.609 & 298.15 & 1600 & 17.081 & $1.739 \mathrm{E}-2$ & $-2.302 E+5$ & $-4.518 \mathrm{E}-6$ & 0.000 & 2939.805 & $0.000 \mathrm{E}+0$ \\
\hline \multirow[t]{2}{*}{$\mathrm{WBr}_{4}$} & g & FactPS & -155000.0 & 456.957 & 298.15 & 900 & 105.213 & $1.287 \mathrm{E}-3$ & $-1.046 E+5$ & $7.512 \mathrm{E}-6$ & 0.000 & 0.000 & $0.000 \mathrm{E}+0$ \\
\hline & & & & & 900 & 2400 & 113.829 & 7.950E-3 & $-5.376 \mathrm{E}+6$ & $-2.492 \mathrm{E}-6$ & 0.000 & 0.000 & $0.000 \mathrm{E}+0$ \\
\hline $\mathrm{Te}_{3}$ & g & FactPS & 203223.0 & 335.942 & 298.15 & 1500 & 58.015 & $4.022 \mathrm{E}-4$ & $-1.428 \mathrm{E}+5$ & $-3.159 E-7$ & 0.000 & 0.000 & $8.504 \mathrm{E}-11$ \\
\hline \multirow[t]{3}{*}{ Te } & g & FactPS & 209451.0 & 182.707 & 298.15 & 500 & 19.397 & $1.837 \mathrm{E}-3$ & $7.991 E+4$ & $0.000 \mathrm{E}+0$ & 0.000 & 0.000 & $0.000 \mathrm{E}+0$ \\
\hline & & & & & 500 & 1000 & 21.873 & $-2.911 \mathrm{E}-3$ & $-4.570 E+4$ & $2.206 \mathrm{E}-6$ & 0.000 & 0.000 & $0.000 \mathrm{E}+0$ \\
\hline & & & & & 1000 & 3000 & 15.280 & $5.442 \mathrm{E}-3$ & $1.156 \mathrm{E}+6$ & $-7.556 E-7$ & 0.000 & 0.000 & $0.000 \mathrm{E}+0$ \\
\hline $\mathrm{TeBr}_{2}$ & g & 5,6 & 14690.0 & 322.185 & 298.15 & 6000 & 58.241 & $-5.021 E-5$ & $-1.598 \mathrm{E}+5$ & $0.000 \mathrm{E}+0$ & 0.000 & 0.000 & $0.000 \mathrm{E}+0$ \\
\hline $\mathrm{Te}_{5}$ & g & FactPS & 220026.8 & 462.011 & 298.15 & 1500 & 107.907 & $3.929 E-4$ & $-2.155 E+5$ & $-3.096 \mathrm{E}-7$ & 0.000 & 0.000 & 8.367E-11 \\
\hline $\mathrm{Br}$ & g & FactPS & 111860.0 & 174.908 & 298.15 & 1300 & 18.547 & $2.582 \mathrm{E}-3$ & $-2.131 E+5$ & $0.000 E+0$ & -49.385 & 2004.288 & $0.000 \mathrm{E}+0$ \\
\hline $\mathrm{HBr}$ & $\mathrm{g}$ & FactPS & -36443.0 & 198.590 & 298.15 & 1800 & 74.218 & $0.000 \mathrm{E}+0$ & $-1.786 \mathrm{E}+6$ & $-9.208 E-7$ & -2213.646 & 30795.695 & $0.000 \mathrm{E}+0$ \\
\hline $\mathrm{Te}_{4}$ & g & FactPS & 217321.9 & 378.983 & 298.15 & 1500 & 82.821 & $7.007 \mathrm{E}-4$ & $-2.418 E+5$ & $-5.506 E-7$ & 0.000 & 0.000 & $1.483 \mathrm{E}-10$ \\
\hline $\mathrm{Br}_{2}$ & g & FactPS & 30910.0 & 245.394 & 298.15 & 1300 & 37.339 & $6.317 \mathrm{E}-4$ & $-1.118 \mathrm{E}+5$ & $0.000 E+0$ & 0.000 & -66.678 & $0.000 \mathrm{E}+0$ \\
\hline $\mathrm{WOBr}_{4}$ & g & 4 & -465290.1 & 468.478 & 298.15 & 6000 & 133.892 & $-1.046 \mathrm{E}-2$ & $0.000 \mathrm{E}+0$ & $0.000 \mathrm{E}+0$ & 0.000 & 0.000 & $0.000 \mathrm{E}+0$ \\
\hline $\mathrm{WO}_{2} \mathrm{Br}_{2}$ & g & 4 & -591203.4 & 380.635 & 298.15 & 6000 & 107.947 & $-8.364 \mathrm{E}-3$ & $0.000 E+0$ & $0.000 \mathrm{E}+0$ & 0.000 & 0.000 & $0.000 \mathrm{E}+0$ \\
\hline $\mathrm{Te}_{6}$ & g & FactPS & 226560.8 & 491.368 & 298.15 & 1500 & 132.869 & 3.529E-4 & $-2.504 E+5$ & $-2.798 \mathrm{E}-7$ & 0.000 & 0.000 & $7.592 \mathrm{E}-11$ \\
\hline $\mathrm{Te}_{7}$ & g & FactPS & 254235.9 & 557.963 & 298.15 & 1500 & 157.770 & $4.434 \mathrm{E}-4$ & $-3.050 \mathrm{E}+5$ & $-3.503 E-7$ & 0.000 & 0.000 & $9.496 \mathrm{E}-11$ \\
\hline $\mathrm{WOBr}_{3}$ & $\mathrm{~g}$ & estimated & -514657.1 & 175.773 & 298.15 & 6000 & 117.922 & $0.000 E+0$ & $0.000 \mathrm{E}+0$ & $0.000 \mathrm{E}+0$ & 0.000 & 0.000 & $0.000 \mathrm{E}+0$ \\
\hline HTe & g & SGPS & 163782.0 & 218.726 & 298.15 & 1300 & 22.839 & $1.561 \mathrm{E}-2$ & $1.911 \mathrm{E}+5$ & $-4.463 \mathrm{E}-6$ & 0.000 & 0.000 & $0.000 \mathrm{E}+0$ \\
\hline \multirow[t]{2}{*}{$\mathrm{WBr}_{5}$} & $\mathrm{~g}$ & FactPS & -199158.0 & 461.302 & 298.15 & 1100 & 132.682 & 3.395E-4 & $-4.961 E+5$ & $0.000 \mathrm{E}+0$ & 0.000 & 0.000 & $0.000 \mathrm{E}+0$ \\
\hline & & & & & 1100 & 2500 & 137.190 & $0.000 \mathrm{E}+0$ & $1.880 \mathrm{E}+6$ & $3.669 \mathrm{E}-7$ & -216.950 & 0.000 & $0.000 E+0$ \\
\hline $\mathrm{WBr}_{3}$ & g & FactPS & 60000.0 & 381.904 & 298.15 & 3300 & 83.137 & $-5.387 \mathrm{E}-4$ & $-2.725 E+5$ & $5.085 \mathrm{E}-7$ & 0.000 & 0.000 & $0.000 \mathrm{E}+0$ \\
\hline $\mathrm{H}_{2}$ & g & FactPS & 0.0 & 130.680 & 298.15 & 1200 & 19.826 & $3.078 \mathrm{E}-3$ & $-2.952 E+5$ & $1.430 \mathrm{E}-6$ & 194.861 & 0.000 & $0.000 \mathrm{E}+0$ \\
\hline $\mathrm{W}_{2} \mathrm{Br}_{6}$ & g & $\begin{array}{c}H \text { from }^{7} \\
S \& C_{P} \text { estimated }\end{array}$ & -234743.3 & 575.006 & 298.15 & 6000 & 182.514 & $2.050 \mathrm{E}-4$ & $-7.414 E+5$ & $0.000 E+0$ & 0.000 & 0.000 & $0.000 E+0$ \\
\hline $\mathrm{TeBr}_{4}$ & $\mathbf{g}$ & 5,6 & -58559.3 & 418.421 & 298.15 & 6000 & 108.156 & $1.004 \mathrm{E}-4$ & $-3.598 E+5$ & $0.000 E+0$ & 0.000 & 0.000 & $0.000 \mathrm{E}+0$ \\
\hline
\end{tabular}


Table 3. Condensed Species used in TRAGMIN $5.1^{8}$

\begin{tabular}{|c|c|c|c|c|c|c|c|}
\hline \multirow{2}{*}{ formula } & \multirow{2}{*}{ state } & \multirow{2}{*}{$T$} & \multirow{2}{*}{$\begin{array}{l}H(T) \text { in } \\
\mathrm{J} \cdot \mathrm{mol}^{-1}\end{array}$} & \multirow{2}{*}{$\begin{array}{c}S(T) \text { in } \\
\mathrm{J} \cdot \mathrm{mol}^{-1} \cdot \mathrm{K}^{-1}\end{array}$} & \multicolumn{3}{|c|}{$C_{p, \mathrm{~m}}=\left(a \cdot T^{0}+b \cdot T^{1}+c \cdot T^{-2}\right)$ in $\mathrm{J} \cdot \mathrm{mol}^{-1} \cdot \mathrm{K}^{-1}$} \\
\hline & & & & & $a \cdot T^{0}$ & $b \cdot T^{1}$ & $c \cdot T^{-2}$ \\
\hline $\mathbf{w}$ & s & 700 & 10196.408 & 54.154 & 23.631 & 0.003611 & 44350.4 \\
\hline $\mathrm{WTe}_{2}$ & s & 298 & -26480.536 & 132.662 & 84.467 & 0.001079 & -707096.0 \\
\hline Te & 1 & 700 & 27668.792 & 95.282 & -34.920 & 0.041919 & 25649593.6 \\
\hline Te & $s$ & 700 & 10702.672 & 71.814 & 39.087 & -0.002674 & -4511607.2 \\
\hline $\mathrm{WOBr}_{2}$ & $\mathbf{s}$ & 298 & -489071.944 & 150.599 & 94.935 & 0.000000 & 0.0 \\
\hline $\mathbf{W}$ & $\mathrm{I}$ & 700 & 62508.960 & 68.308 & 23.631 & 0.003611 & 44350.4 \\
\hline $\mathrm{WOBr}_{3}$ & s & 298 & -557325.536 & 192.472 & 119.922 & 0.000000 & 0.0 \\
\hline $\mathrm{Te}_{2} \mathrm{Br}$ & $\mathbf{s}$ & 298 & -48187.128 & 147.432 & 62.057 & 0.050865 & 0.0 \\
\hline $\mathrm{WOBr}_{4}$ & $s$ & 298 & -588308.056 & 259.425 & 163.193 & -0.010493 & 0.0 \\
\hline $\mathrm{WBr}_{4}$ & $s$ & 700 & -209555.640 & 345.042 & 123.131 & 0.029727 & 836.8 \\
\hline $\mathrm{WO}_{2}$ & $s$ & 700 & -562756.368 & 106.679 & 74.797 & 0.005895 & -2579436.0 \\
\hline $\mathrm{WBr}_{5}$ & $s$ & 298 & -311708.000 & 271.960 & 125.863 & 0.100073 & 22175.2 \\
\hline
\end{tabular}

Table 4. Gaseous species used in TRAGMIN $5.1^{8}$

\begin{tabular}{|c|c|c|c|c|c|c|c|}
\hline \multirow{2}{*}{ formula } & \multirow{2}{*}{ state } & \multirow{2}{*}{$T$} & \multirow{2}{*}{$\begin{array}{l}H(T) \text { in } \\
\mathrm{J} \cdot \mathrm{mol}^{-1}\end{array}$} & \multirow{2}{*}{$\begin{array}{c}S(T) \text { in } \\
\mathrm{J} \cdot \mathrm{mol}^{-1} \cdot \mathrm{K}^{-1}\end{array}$} & \multicolumn{3}{|c|}{$C_{p, \mathrm{~m}}=\left(a \cdot T^{0}+b \cdot T^{1}+c \cdot T^{-2}\right)$ in $\mathrm{J} \cdot \mathrm{mol}^{-1} \cdot \mathrm{K}^{-1}$} \\
\hline & & & & & $a \cdot T^{0}$ & $b \cdot T^{1}$ & $c \cdot T^{-2}$ \\
\hline $\mathrm{Te}_{2}$ & g & 700 & 177786.528 & 292.018 & 54.225 & -0.006251 & -3995720.0 \\
\hline $\mathbf{N}_{2}$ & g & 700 & 11941.136 & 216.869 & 28.125 & 0.004937 & -411287.2 \\
\hline $\mathrm{WBr}_{4}$ & g & 700 & -111871.792 & 548.296 & 104.039 & 0.010828 & -1095371.2 \\
\hline $\mathrm{Te}_{3}$ & g & 700 & 226312.560 & 384.907 & 58.216 & -0.000008 & -160665.6 \\
\hline Te & g & 700 & 217806.488 & 200.451 & 18.046 & 0.002607 & 474465.6 \\
\hline $\mathrm{TeBr}_{2}$ & g & 298 & 14690.024 & 322.185 & 58.241 & -0.000050 & -159828.8 \\
\hline $\mathrm{Te}_{5}$ & g & 700 & 263027.160 & 553.221 & 108.102 & -0.000008 & -232630.4 \\
\hline $\mathrm{Br}$ & g & 700 & 120223.056 & 192.774 & 18.903 & 0.002238 & 221752.0 \\
\hline $\mathrm{HBr}$ & $\mathrm{g}$ & 700 & -24560.080 & 223.865 & 27.263 & 0.005301 & -271123.2 \\
\hline $\mathrm{Te}_{4}$ & g & 700 & 250228.304 & 448.851 & 83.165 & -0.000017 & -272796.8 \\
\hline $\mathrm{Br}_{2}$ & g & 700 & 45768.776 & 276.872 & 37.267 & 0.000657 & -131796.0 \\
\hline $\mathrm{WOBr}_{4}$ & $\mathrm{~g}$ & 298 & -465290.088 & 468.587 & 133.892 & -0.010464 & 0.0 \\
\hline $\mathrm{WO}_{2} \mathrm{Br}_{2}$ & $\mathrm{~g}$ & 298 & -591203.384 & 380.744 & 107.947 & -0.008364 & 0.0 \\
\hline $\mathrm{Te}_{6}$ & $\mathrm{~g}$ & 700 & 279516.304 & 603.818 & 133.043 & -0.000008 & -265684.0 \\
\hline $\mathrm{Te}_{7}$ & g & 700 & 317105.360 & 691.439 & 157.988 & -0.000013 & -324260.0 \\
\hline $\mathrm{WOBr}_{3}$ & g & 298 & -514657.104 & 175.883 & 117.922 & 0.000000 & 0.0 \\
\hline HTe & $\mathrm{g}$ & 700 & 175987.408 & 244.584 & 30.577 & 0.004443 & -861485.6 \\
\hline $\mathrm{WBr}_{5}$ & g & 700 & -146728.696 & 572.505 & 132.616 & 0.000389 & -480323.2 \\
\hline $\mathrm{WBr}_{3}$ & $\mathrm{~g}$ & 700 & 92830.408 & 451.600 & 82.253 & 0.000732 & -152716.0 \\
\hline $\mathbf{H}_{2}$ & $\mathrm{~g}$ & 700 & 11748.672 & 155.607 & 24.577 & 0.004925 & 705004.0 \\
\hline $\mathrm{W}_{2} \mathrm{Br}_{6}$ & $\mathrm{~g}$ & 298 & -234743.320 & 575.116 & 182.514 & 0.000205 & -741404.8 \\
\hline $\mathrm{TeBr}_{4}$ & g & 298 & -58559.264 & 418.530 & 108.156 & 0.000100 & -359824.0 \\
\hline
\end{tabular}




\section{References:}

(1) Bale, C. W.; Bélisle, E.; Chartrand, P.; Decterov, S. A.; Eriksson, G.; Gheribi, A. E.; Hack, K.; Jung, I.-H.; Kang, Y.-B.; Melançon, J.; Pelton, A. D.; Petersen, S.; Robelin, C.; Sangster, J.; Spencer, P.; Ende, M. Van. FactSage Thermochemical Software and Databases , 2010 - 2016. CALPHAD Comput. Coupling Phase Diagrams Thermochem. 2016, 54, 35-53. https://doi.org/10.1016/j.calphad.2016.05.002.

(2) Oppermann, H.; Kunze, G. WOBr3, WOBr2-Bildungsenthalpie Und Thermische Zersetzung. Zeitschrift fur Anorg. und Allg. Chemie 1972, 387, 329-338.

(3) Haag, J.; Alpen, U. V.; Gmelin, E.; Rabenau, A. Electrochemical and Specific Heat Measurements on Tellurium-Halogen Systems. Zeitschrift fur Naturforsch. - Sect. A J. Phys. Sci. 1979, 34, 969-975. https://doi.org/10.1515/zna-1979-0807.

(4) Oppermann, H.; Stöver, G. Sättigungsdrücke, Bildungsenthalpien von WOBr4 Und WOBr2, Die Reaktionsgleichgewichte $2 \mathrm{WO} 2 \mathrm{Br} 2=\mathrm{WO} 3+\mathrm{WOBr} 4 \mathrm{Und} 2 \mathrm{WOBr} 4+\mathrm{SiO} 2=\mathrm{WO} 2 \mathrm{Br} 2+\mathrm{SiBr} 4$. Zeitschrift fur Anorg. und Allg. Chemie 1971, 383, 14-28.

(5) Oppermann, H.; Kunze, G.; Wolf, E.; Kokovin, G. A.; Sitschova, I. M.; Osipova, G. E. Untersuchungen Zum System Te/O/I. Z. anorg. allg. Chem. 1980, 461, 165-172. https://doi.org/10.1002/zaac.19804610125.

(6) Oppermann, V. H.; Titov, V. A.; Kunze, G.; Wolf, E. Untersuchungen Rum System Te/O/Br Von. Zeitschrift fur Anorg. und Allg. Chemie 1978, 439, 13-28.

(7) Kaposi, O.; Popović, A.; Marsel, J. Mass Spectrometric Studies of Tungsten Bromides and Oxybromides. J. Inorg. Nucl. Chem. 1977, 39, 1809-1815. https://doi.org/10.1016/0022-1902(77)80206-6.

(8) Krabbes, G.; Bieger, K.Sommer, H.; Söhnel, T.; Steiner, U. GMIN 5.1: Package TRAGMIN for Calculations of Thermodynamic Equilibrium. Dresden 2014. 